\title{
An Overview of the Nineteenth and Twentieth Centuries' Theories of Light, Ether, and Electromagnetic Waves
}

\author{
Salvo D'Agostino \\ Università "Sapienza", Rome, Italy \\ Email: Saldagostino21@gmail.com
}

Received 13 January 2016; accepted 13 February 2016; published 16 February 2016

Copyright (C) 2016 by author and Scientific Research Publishing Inc.

This work is licensed under the Creative Commons Attribution International License (CC BY).

http://creativecommons.org/licenses/by/4.0/

c) (i) Open Access

\begin{abstract}
Johannes Kepler, the seventeenth century celebrated astronomer, considered vision as the effect of its alleged cause-the Lumen. Since many centuries, scientists and philosophers of Light were especially interested in theories and experiments on the cause-effect relationship between our vision and its alleged cause. But the Nineteenth and Twentieth Centuries' contributions of Helmholtz, Maxwell, Hertz, and Lorentz, proved that Light was an electromagnetic wavelike phenomenon, which propagated trough space or ether by an exceptionally high velocity. In my paper I analyze some of the reasons that might justify the controversies among the major experts in Physics and Electrodynamics. In 1905 Albert Einstein found that abolishing Ether would remarkably improve his new Special Relativity theory, and Maxwell's and Hertz's Electrodynamics. His theory was accepted by a large majority of physicists, Max Planck included, but he also found a ten-year silence on the side of Poincaré, and moderate oppositions from Lorentz, the great expert in classical Electrodynamics.
\end{abstract}

\section{Keywords}

Einstein, Maxwell, Helmholtz, Hertz, Lorentz, Velocity of Light

\section{Introduction}

Johannes Kepler studied what he considered the external cause of vision, the Lumen ${ }^{1}$. Kepler's view of Lumen as a Light's material support represented the birth of a new paradigm on the existence of various levels of Light's supporters. In his 1611 Dioptrics Kepler's theory of vision was based on the concept of rays propagating

${ }^{1}$ Ronchi, V (1952). 
from luminous and illuminated object. The theory is still accepted as an elementary theory, although Kepler prudently admitted the difficulty of explaining virtual images, and he limited his approach to the study of white light. Kepler's overthrow of the Middle Age conception of Fire as the unique supporter of Light was also confirmed by Galileo's observations with his telescope ${ }^{2}$. Huygens's and Newton's opposite views on the essence of Light concerned a distinction between particles and waves. In the 1820s, Augustine Fresnel was convinced that Light was a transversal wave, and its source was in the Ether, a Lumen very much dissimilar from ordinary materials. An analogy with acoustics and sound was the implicit model because a vibrating string was the causal source of music and sounds. But, the source of Light cannot be assimilated to the string elasticity because the Light's velocity was incommensurably higher than any terrestrial velocity. Maxwell' field theory of electromagnetic waves included an electromagnetic theory of Light, but it owed its success to M.'s recourse to the action at a distance theory of Karl Fredrik Gauss and Wilhelm Weber, clearly opposing his electromagnetic field program. Ten years after Maxwell, Heinrich Hertz performed the first controlled production of electromagnetic waves.

The Discovery of $\mathrm{X}$ rays extended the concept of something later on identified as of very high frequency electromagnetic waves. But the spirit of a glorious science did not last for more than a few years: who could have imagined that the very assertor of an indubitable truth in scientific law, the great Max Planck, was destined to contradict Maxwell and Hertz on the wavelike nature of Light? It is well known that Planck discovered that a Light wave included energy packets, the today well known Photons.

In my paper I am concerned with Nineteenth Century's contributions to theories on the velocity of Light by Maxwell, Hertz, Lorentz and Helmholtz. I underline a comparison between Hertz's reflective criticism on his conception of the a-priory assumption of physics theory, and, on the other side, Lorentz's and Zeeman's justifications for the success of their empirically well supported theory. Following the abolition of ether in his celebrated 1905 essay on Special Relativity, Einstein turned many times, and in the majority of his essays, to reconsider the ether's role in physics and concluded his reflections with the admission of the indispensability of ether in his General Relativity Theory.

\section{Maxwell's Theory of Light: A Structural Similarity between Maxwell's Electromagnetic Waves and the Waves of Light}

James Clerk Maxwell brought into his Electromagnetic Theory of Light concepts and experimental data obtained from radically different atomistic and field theories. Theories of Mechanics and Electrostatic Potentials, and Wilhelm's Weber's outstanding contributions to Electrodynamics were largely utilized. Green's and Stokes's elastic and hydrodynamic theories were also the underlying mathematics of M.'s equations for the velocity of electric and magnetic waves. Identification of this velocity with the then known value for the velocity of light, represented the transition from statics to dynamics field theory, a topic which was almost an exclusive merit of the Scottish scientist. It required however a very hard approach, that included elastic, hydrodynamic, and Lagrangian theories.

Maxwell's contributions to Electromagnetism and Field Theory have been frequently discussed in the history of science literature, with special attention to his original discovery of his celebrated equations ${ }^{3}$. However, to my present knowledge, the connection in Maxwell's research between velocity of his electromagnetic waves and Weber's velocity has not been analyzed, although this is one of the characteristic feature of Maxwell's approach to his theory of Light. The quasi equality between Weber's velocity of motion of electric masses and Maxwell's velocity of electromagnetic waves has been misinterpreted as a logical consequence of premises that were foreign to Maxwell. Maxwell in fact duly underlined Weber's systematic definition of absolute units for electric and magnetic units, although he refused Weber's conception of a velocity of electric masses and only accepted the experimental evidence of Weber's achievements. However, he considered the quasi-equality of Weber's velocity with his electromagnetic waves velocity one of the most important pieces of evidence in favor of his electromagnetic theory of light ${ }^{4}$.

The Light velocity in ether was, however, dependent from the choice of Weber's Electrodynamics units, and, in order to meet this problem M. introduced an ad hoc system of three electric and magnetic units. At the end of

\footnotetext{
${ }^{2}$ Bartellini, F. (2010).

${ }^{3}$ Hendry, J. (1986).

${ }^{4}$ D’agostino, S. (1996): pp. 5-51.
} 
the Nineteenth Century, the Italian Giovanni Giorgi enlarged the three units system in a more consistent four units system. Maxwell's difficulties with this problem, and his struggle to find arguments in support of his system, are significantly documented in his various attempts to connect Weber's factor to the constants of the Ether, a necessary prerequisite to reach the conclusion that the same factor represented the velocity of electromagnetic waves in Ether $^{5}$.

In 1862, he introduced an ad hoc argument from elasticity in order to demonstrate that Weber's factor was the velocity of Light. It is therefore understandable that his later derivation in 1873 from purely metrological arguments of the same demonstration, served to settle the implicit charge of a theoretical circularity ${ }^{6}$. The special form of Maxwell's 1873 demonstration of Weber's equality, based on metrological arguments, afforded a decisive step in the direction of freeing M.'s theory from its early connections with the elastic ether. In fact, let us notice Maxwell's comment in his paper on ether ${ }^{7}$ : “....the only ether which has survived is that which was invented by Huygens to explain the propagation of light”. And he added: “...the best way to compare the properties of the electromagnetic medium to that of Light is to compare the two velocity... In the form which treats the phenomena of light as the motion of an elastic solid, the wavelike theory is still encumbered with several difficulties”.

Although harshly criticized from his contemporaries, M.'s unusual approach relating the velocity of Light to Weber's' factor ratio promoted interesting developments. Between 1900 and 1905, Lord Rayleigh applied with success dimensional analysis to problems of mechanics, heat transfer, optics and electromagnetism. In 1914, E. Buckingham generalized M.'s theorems of products with fixed dimensions in a theory of physically similar systems, the so-called " $\Pi$ theorem", through which dimensional analysis a useful tool in the theory of physical similitude and of physical models ${ }^{9}$.

Writing in 1893, twenty years after M.’s Treatise, Oliver Heaviside grasped the significance of Maxwell's innovations: "Elastic solid theories are a great deal too precise in saying what light consists of, and mechanical speculations in general should be received with much caution, and regarded rather as illustrations or analogies than expressions of facts. We do not know enough yet about the ether for dogmatizing." 10 The essence of Heaviside's statement concerns changes in "permittivity" and "electrical eolotropy". They can be independently observed, i.e., observed through electrical esperiments which do not imply elastic-optical concepts or theories, whereas assertions concerning properties like density and elasticity of ether cannot be observed. The former are observable (factual) properties while the latters remain hypothetical assumptions.

\section{A Phenomenological Foundation for an Objective Theory of Light in Helmholtz's Parallelism of Regularities}

Hermann von Helmholtz's (1821-1894) contributions to an epistemological foundation of a theory of vision represent a remarkable part of his work on the Kantian problem of the a-priori in physics ${ }^{11}$. Kant did not excerpt his influence on Helmholtz through the mediation of the romantic philosophy of Shelling and Schopenhauer, less then ever through the Hegelian Naturphilosophie, which Helmholtz vehemently contested. He was inspired by Johannes Müller's theory of the specific energy of "sensory nerves": "The stimulation of the optic nerve produces only sensations of light whether that stimulation be caused by objective light (vibrations in the ether), by an electrical current through the eye, by pressure on the eyeball, or by rapid directional changes of the eye". ${ }^{12}$

By following Mueller's theory, Helmholtz deeply modified the common naive view of perceptions as an image of external influences, such as the Keplerian Lumen. He denied that our senses are passive receptors of a supposedly faithful image of the world: "Inasmuch as the quality of our sensations gives us a report of what is peculiar to the external influence by which it is excited, it may count as a symbol of it, but not as an image. For from an image one requires some kind of alikeness with the object of which it is an image". ${ }^{13}$

His "report" type of perception did not prevent however that Helmholtz found a sort of a likeness of percep-

\footnotetext{
${ }^{5}$ Ibid, 5-51.

${ }^{6}$ Ibid, 45.

${ }^{7}$ Maxwell, J.C. (1954): pp. 763-775.

${ }^{8}$ Maxwell J.C. (1954): Vol.2, p. 772.

${ }^{9}$ D’Agostino, S. (1996): p. 40.

${ }^{10}$ Hunt (1991): pp. 231-232.

${ }^{11}$ Hermann von Helmholtz (1977).

${ }^{12}$ Hermann von Helmholtz (1977): p. 119.

${ }^{13}$ Hermann von Helmholtz (1977): pp. 121-122.
} 
tions with the object of science, because in his view "every law of nature” is founded in general on a "parallelism of regularities": "Every law of nature asserts that upon preconditions alike in a certain respect, there always follow consequences which are alike in a certain other respect. Since like things are indicated in our world of sensations by like signs, an equally regular sequence will also correspond in the domain of our sensations to the sequence of like effects by law of nature upon like causes". ${ }^{14}$

Helmholtz believed that his "parallelism of regularities" view of scientific knowledge brought the fullest confirmation of Kant's a-priori doctrine ${ }^{15}$. Let us devote a few lines to illustrate Helmholtz' original view of Kant, because it somehow modified "Kant's doctrine”. He added to it a distinction, or resolution, of the forms of intuition into two specific features: a general form and a narrower specification. What was truly a-priori in every sensory perception was a general form devoid of any content, as exemplified in the spatial perception of place, or, in the visual perception as an aggregate of colored surfaces, which always compose our visual field ${ }^{16}$ :

"Everything our eye sees, is an aggregate of colored surfaces in the visual field-that is our \{general \} form of intuition. The particular colours, which appear on this or that occasion -their arrangement and sequence -, this is the result of external influences and is not determined by any law of our makeup."17.

Let us remark that Helmholtz's study of perceptions as distinguished from a merely linguistic approach, opened a new field of inquiry, that still bears fruit in modern research.

Applying to the study of perceptions in general his distinction of the Kantian a-priori forms in spatial perceptions, Helmholtz found that between the various kinds of sensations there exist differences in the form of two neatly distinguished types: one form concerned sensations belonging to different senses, such as sight and sound, the other to a specific sense as sight; he called this a difference in the modality (of sensations). Inaccessible to Kant's linguistic distinctions, the modality distinction was accessible only via an operational approach ${ }^{18}$. Let us remark that Helmholtz's study of perceptions as distinguished from a merely linguistic approach, opened a new field of inquiry, that still bears fruit in modern research.

On occasion of the anniversary celebration at the Berlin University in 1878, Helmholtz delivered an Address, published by the title “The Facts in Perception"19 ("Die Tatsachen in die Vahrnemung”). He turned his attention to the problem of the correlation between the presumed external agent of Light and the observable effects on our sensation $^{20}$. The problems evidently concerned the old fashioned Keplerian Lumen-Light correlation. In a modern terminology this correlation can be interpreted in a double sense: either in the sense that an ideal theory should achieve a perfect adequacy between concepts and the corresponding perceptions, the Helmholtz' "parallelism”, or in the more naturalist view, the existence of ether ${ }^{21}$.

Let us look for a possible interpretation at Moritz Schlick's detailed study of Helmholtz's papers. According to Schlick, Helmholtz supported a physiological and psychological interpretation of those forms of intuition that Kant had assigned to a transcendental aspect of knowledge. ${ }^{22}$ I argue that by limiting the transcendental character of the Kantian a priori forms of intuition, Helmholtz diminished their cognitive impact, and thus contributed,

\footnotetext{
${ }^{14}$ Hermann von Helmholtz (1977): p.122.

${ }^{15}$ Ibid: pp. 118-119.

${ }^{16}$ Ibid: p. 121.

${ }^{17}$ Ibid: pp. 119. Notice that "laws of our makeup" is the English wording or the German "a-priory form of intuition".

${ }^{18}$ The assertion that Helmholtz supported an a-priory Euclidean geometry, thus contradicting his well cknown adhesion to Riemann, was the false topic of some bibliography. In order to disprove the above assertion let me quote just his passage: "the axioms of Euclidean geometry represent thus the intuition's narrower specification and thus space three-dimensionality represents its empirical specification. In short, a form of intuition which limits our perception of space to its three-dimensional formulation, is too narrow (too full of a particular content) for representing all possible contents of our experience and, as such, cannot be a truly a-priori form”.

${ }^{19}$ Hermann von Helmholtz (1977): pp. 115-163.

${ }^{20}$ D’Agostino, S. (2005). Let us remark that in his neo-Kantian approach to knowledge, Helmholtz trusted to a "parallelism of regularities" the support for his view of scientific realism. In the above situation, he was mainly concerned to defending the objectivity of science from Ernst Mack's attach, whereas in a different situations he intended to defend his phenomenological position from a crude realistic attach by scientists themselves.

${ }^{21}$ At the close of his Address, Helmholtz surprisingly emphasized that his favorite approach to an ideal theory had no less than Goethe and Kirchhoff as its supporters. He agreed with the poet that one should simply demand from science that it should be only an artistic arrangement of the facts and form no abstract concepts going beyond this:

"I consider it a propitious sign that Goethe, both here and in other matters, finds himself with me on the same path. His theory of colors can be considered an attempt to save the immediate truth of sensory impressions from attaches by science.”[Italics S.D.]

${ }^{22}$ Hermaann von Helmholtz (1977). Introduction and Bibliography by Cohen, R. S., \& Elkana, Y. 177-178.
} 
so-to-speak, to their "secularization.” Helmholtz's use of the term "description” referred only to physical phenomena and not to the presumed underlying agents of our perceptions, as the Keplerian Lumen. Notice however that he intended to defend the immediate truth of sensory impressions from attaches by science. Ernst Cassirer rightly considered Helmholtz an important representative of the German phenomenological tradition in physics $^{23}$.

\section{Heinrich Hertz: Ether Polarization Confirms Faraday's View of an Independent Existence of Forces in Space}

One can find agreement and continuity between Helmholtz's and Hertz's theories and conceptions ${ }^{24}$, but the historian discovered that their epistemologies present remarkable notes of discordance. In his preface to Hertz's Principles of Mechanics of 1894, in a passage often ignored in the historical literature, Helmholtz inserted a clear statement on the difference between his and Hertz's epistemologies ${ }^{24}$. He remarked that his favorite student had adopted mechanical representations instead of the "simple representation of physical facts and laws by "systems of differential equations," the latter being the phenomenological approach that Helmholtz had embraced $^{25}$.

Let us explore those features that could possibly find some ground for his master's charge. Since his initial approach to his experiments, Hertz soon realized that the physical basis of Helmholtz's Electrodynamics did not comport waves, because it was fundamentally an action-at-a-distance theory. In Helmholtz's Electrodynamics in fact, propagation in a medium was considered as a second order effect, which could prevail by a mathematical passage in the so called limiting case, only to comply with some results of Maxwell's theory. In Hertz's criticism, Helmholtz supported a theory of waves as polarization of dielectrics, in the sense of the Poisson-Mossotti polarization theory ${ }^{26}$, a peculiar blending of action-by-contact in dielectrics and action-at-a-distance in an ether-less vacuum. Hertz' conception is clearly on the opposite: ether is an-priory concept, indispensable for propagation. Another conceptual obstacle, and a second source of difficulties for Hertz's experiment was his doubt that he did "not always felt quite certain of having grasped the physical significance of his (i.e. Maxwell's) statements". It can be argued that some obscurities were in Maxwell's papers, but the historian can now argue that the main obstacle was Helmholtz: what actually Hertz initially knew on M. theory of Light was mainly derived from Helmholtz' theory.

In my analysis, I find enough evidence for proving that the most relevant topic for Hertz's experimental success was his original conception of ether as a primitive polarized medium ${ }^{27}$. He drastically refuted in fact Helmholtz's thesis that ether was secondarily connected to polarizations of the "Poisson type" (Hertz: “...it is quite another question...”). Hertz suddenly understood that the polarized medium was no less than ether itself, i.e., ether coincided with a polarized dielectrics. If this thesis is accepted, one has an explanation of the otherwise obscure passage in Hertz's Introduction: “I have rather been guided (in my experiments) by Helmholtz's work, as indeed may plainly be seen from the manner in which the experiments are set forth. But unfortunately, in the special limiting case of Helmholtz's theory which leads to Maxwell's equations, and to which the experiments pointed, the physical basis of Helmholtz's theory disappears, as indeed it always does [in Helmholtz] as soon as action-at-a-distance is disregarded. I therefore endeavored to form for myself in a consistent manner the necessary physical conceptions, starting from Maxwell's equations" ${ }^{, 2}$. The different meaning attributed by Hertz to a polarization theory of ether can be better understood in the light of his conclusions on the nature of electric force, at the end of his work in electromagnetism. His basic tenet was in fact, that air and empty space could

\footnotetext{
${ }^{23}$ Cassirer, E. (1950).

${ }^{24}$ Hertz, H. (1956a).

25،"English physicists, e.g. Lord Kelvin in his theory of vortex-atoms, and Maxwell, in his hypothesis of systems of cells with rotating contents, on which he bases his attempt at a mechanical explanation of electromagnetic processes--have evidently derived a fuller satisfaction from such explanations than from the simple representation of physical facts and laws in the most general form, as given in systems of differential equations. For my own part, I must admit that I have adhered to the latter mode of representation [by systems of differential equations] and have felt safer in so doing; yet I have no essential objections to raise against a method which has been adopted by three physicists of such eminence” (Hertz, H., 1956a). (Prefase by H. Von Helmholtz: not numbered).

${ }^{26} \mathrm{Helmholtz}$ 's theory treated polarization as the effect of the bound charge, the same charge that in its free state was responsible for the conduction current. In this last sense, I think, we must understand Helmholtz's statement that "air and empty space behave like all other dielectrics", the statement that Hertz calls his rejected third hypothesis. Helmholtz meant that air is polarizable "a la Poisson", but polarization of this type is, in air, very low in comparison with other material dielectrics. Hertz did not search in his experiment for a Helmholtz's polarization.

${ }^{27}$ D’Agostino, S. (1975): p. 294.

${ }^{28}$ Hertz, H. (1962): p. 27.
} 
support electromagnetic wave not for the reason that they participate in the action of the supposed bound charges-according to a Poisson-Helmholtz conception, but, just on the reverse, air and material dielectrics behave like empty space for the reason that they participate in the nature of empty' space, -i.e. they embed "ether”. For the electric force is, essentially, for Hertz a polarization of Ether ${ }^{29}$. One year later, following his experiments, he will attribute to Faraday's glory the new revolutionary concept of a dielectric action, and will express more clearly his ideas on this matter:'The most direct conclusion of the experiment on the finite velocity of propagation of electromagnetic forces, is the confirmation of Faraday's view, according to which the electric forces are polarizations existing independently in space”.

A deeper consideration of the process which is at the origin of this Hertz's contribution must take into account, in my opinion, Hertz's first approach to Maxwell's theory in his 1884 theoretical paper ${ }^{30}$ : Maxwell's equations are therein deduced from told electrodynamics and from hypotheses that did not imply a dielectric action, in Helmholtz sense. Maxwell's equations result from a combination of Faraday's induction law, energy conservation, and his "principle of the unity of fields", a reiteration process, mathematically equivalent to a series expansion of the field. This fact might have counted in favor of his considering electric waves as dependent on something more essential than the behavior of material dielectrics. An important point in this paper is the so-called "principle of the unity of fields": the electric field has the same nature, irrespective of the mechanism through which it is generated, be it by standing or moving charges or by a static or changing magnetic field. The justification is found in the fact that “... the electric field, according to Faraday's conceptions, is something existing in itself in space independently of the way in which it is generated”. It is this physical conception of a field as a self-sustaining entity that makes possible the conception of a self-sustaining wave of electric force as a physical entity (not a purely mathematical one). Hertz's conceptions at this turning point are also manifested by a modification in his outlook upon experiments: he now becomes aware that he is looking for waves of electric force in air. As a consequence, some observations that were disregarded in the past as meaningless became relevant from his new outlook. A further example is given by Hertz in another passage:"Somewhat later on, I thought that I noticed a peculiar reinforcement of the action in front of such shadow forming masses, and of the walls of the room. At first it occurred to me that this reinforcement might arise from a kind of reflection of the electric force from the conducting masses; but although I was familiar with the conceptions of Maxwell's theory, this idea appeared to me to be almost inadmissible so utterly was it at variance with the conceptions then current as to the nature of an electric force". Notice the lack of relevance in observations, when the proper theoretical framework is missing.

As regards Hertz' new conception of electric forces, let us notice that Hertz drastically refuted Helmholtz' atomistic view of electric and magnetic forces. Hertz firmly believed that atoms were in no wise fit for a logically clear and secure foundation of a mathematical theory of physics ${ }^{31}$. In his 1894 Prinzipien, he discussed the problems of atoms in connection with his discussion of Energetics and of its fitness to represent a suitable image (Bild) of physics. He favored Energetics because "it avoids the stumbling-blocks which endangered the appropriateness of the first \{image\}". i.e., An image (Bild) that represented reality in terms of forces acting between atoms and molecules it is unfit "to serve as a known and secure foundation for mathematical theories". In Hertz's view, “the arbitrary assumed properties of the atoms may not affect the final results, \{because $\}$ the results may be correct. Nevertheless the details of the deduction are in great part presumably false; the deduction is only in appearance a proof. The earlier modes of thought in physics scarcely allowed any choice or any way of escape”.

Overcoming the probable surprise of the modern physicist, let us try to translate Hertz's statements into a modern interpretation: it is impracticable to fit mathematical equations with the types of hypotheses that could represent minute structural properties of matter. Taking account of Hertz's conception of theory and of his views on the mathematics-physics relationship, this means that the form itself of the mathematical structure of Hertz's electrodynamics made impossible its fitting, and on a rational ground, with the atomist hypothesis. In this view, Hertz somehow reflected Maxwell's general ideas on the inconclusiveness of experimental confirmation of "the arbitrary assumed properties of the atoms" ${ }^{32}$.

As regards Hertz's views on the nature of his electromagnetic waves, let us notice that his 1884 derivation of

\footnotetext{
${ }^{29}$ Hertz, H. (1962): p. 27.

${ }^{30}$ Hertz, H. (1956b): pp. 273-290.

${ }^{31}$ Hertz, H. (1962).

${ }^{32}$ Hertz, H. (1956b): p. 35.
} 
M.'s equations, implying the unification of ether with dielectric polarization, and with electricity itself, represented an original initial solution to the problem of the electromagnetic waves physical nature. In fact, Max Planck believed ${ }^{33}$ that Hertz's original approach to Maxwell was very worthy of physical and epistemological attention.

\section{Lorentz' Ether: The Last Resort for an Absolute Velocity of Light}

In 1895, Heindrik Antoon Lorentz published his fundamental work "Versuch einer Theorie der electrischen und optischen Erscheinungen in bewegten Körpern”, that included his researches on the optical and electromagnetic effects of dielectrics in motion treated from a unitary point of view. It is worth to remark that he learned his electromagnetism from Helmholtz's hands. For the first time, Maxwell's macroscopic equations were derived in terms of average values of microscopic fields, thus showing that the micro-macro connections were feature of a good theory. In this work, Lorentz called the microscopic charges: "ions”, but changed this term to that of "electrons" in 1899, after the 1897 discovery of the particle by J.J. Thomson. From the polarization of the outer lines of Zeeman triplet, he predicted the negative charge of the "Lightion”, thus correcting Zeeman's initial conclusion in 1897 of a positive charge. Due to the experimental evidence, Lorentz was perhaps encouraged to ignoring Maxwell's and Hertz's objections, and included his theory of electrons in those molecular and atomic theories that were the targets of the two scientists. To their criticism he opposed the consideration that "the too adventurous physicists, although they may run the risk of being deluded by false prospects, are very often repaid by the boldness of molecular hypotheses" ${ }^{\text {,3 }}$. Although he claimed that his electron theory was an offspring from the great theories of electricity of Faraday and Maxwell, he also underlined the differences. "You all know this theory of Maxwell, which we may call the general theory of the electromagnetic field, and in which we constantly have in view the state of the matter of the medium by which the field is occupied. While speaking of this state, I must call your attention to the curious fact that, although we never loose sight of it, we need by no means go far in attempting an image of it and, in fact, we cannot say much about it. It is true that we may represent to ourselves internal stresses existing in the medium surrounding an electrified body or a magnet that we may think of electricity as of some substance or fluid, free to move in a conductor. Many physicists have done all this and Maxwell himself has set the example. Yet, it must not be considered as really necessary; we can develop the theory to a large extent and elucidate a great number of phenomena, without entering upon speculations of this kind"34.

Lorentz's objected that "the theory of electrons is to be regarded as an extension to the domain of electricity of the molecular and atomic theories that have proved of so much use in many branches of physics and of chemistry. Like these, it is apt to be viewed un favorably by some physicists who prefer to push their way into new and un explored regions by following these great highways of science which we possess in the laws of thermodynamics”.

He justified his method on the ground of a new role of mathematics in physics, and expresses his belief a few lines afterwards: "the mathematical relations have thus come to take a very pre-eminent place".

As often in physics, the ground of his ideas found support in another tradition, that of Hermann Helmholtz and Wilhelm Weber's electrodynamics, a paradigm alternative to that of Maxwell and Hertz. In his 1878 essay: "Concerning the Relations between the Velocity of Propagation of Light and the Density and Composition of Media”, he supported Hertz's view of ether, whereas the Poisson type dielectric holds only in ponderable bodies. His radical thesis on the null variation of the Light's velocity relative to the earth's motion is presented in his 1887 essay: "De influence du movement de la terre sur les phenomènes lumineux". The negative result of the Michelson-Morley experiment is justified by the theory of a compensatory effect of a Fitzgerald contraction and an account of an apparent time variation. In an 1891 essay “Electricitet en Ether”, taking account of Hertz's experiment, he attempted a conciliation between Helmholtz's and Maxwell's electromagnetism.

His new theoretical approach is presented in his great 1892 Memoire "La theorie électromagnetique de Maxwell et son application aux corps mouvantes”. A remarkable balance between a physical electrostatic contraction and the Lorentz transformation of Maxwell's equations explains the Michelson Morley null result. The composite aspect of Lorentz's theory could have appeared a compromise between the pure field theories of Maxwell and Hertz, and the particulate theories of Weber and Helmholtz. But it was a rather elaborated theory, although

\footnotetext{
${ }^{33}$ Planck, M. (1931).

${ }^{34}$ Lorentz (1952).
} 
it was soon superseded by Einstein's relativistic Electrodynamics.

The conceptual relevance of theoretical debates among three great expert of the end of century Electrodynamics, however, cannot ignore or understate the sometime decisive role of experiments in their role of deciding between alternative positions. I believe that the most important piece of information for the construction of microphysics, alternative to Maxwell's and Hertz's, and to Lorentz' theory, was Pieter Zeeman's amazing prediction in August 1896 that the oscillating particles emitting light had a subatomic mass. An historical and epistemological interest is therefore presented by Zeeman 1902 Nobel Lecture description of the story of his discovery of the Zeeman effect ${ }^{35}$. As Zeeman tells in his Lecture, on the story of his first approach to the 1896 experiment, he was trying to obtain the effect just by relying on the greater resolution of his spectroscope, a Rowland concave reflection grating, improved by screening the source with a slit. In spite of these instrumental improvements, the result was very uncertain because, after the magnet was powdered, the sodium line was not split but just slightly widened (two tenths of an Angstrom) and this effect could not be unambiguously explained in terms of the magnetic force because thermodynamic effect could also have contributed to it. Zeeman was very dubious of the interpretation of the result since Maxwell's authoritative pronouncement against a positive result was in the back of his mind. But he changed his expectations on the splitting of the lines, explained it through Lorentz's particulate hypothesis, confirmed the predicted new type of splitting (a doublet) in the direction of the magnetic force, and checked that their polarization was as the theory predicted. Perhaps the most important piece of information for the construction of microphysics was Zeeman's amazing prediction in August 1896 that the oscillating particles emitting light had a subatomic mass. He thus strongly contributed to the discovery of the first subatomic particle. In 1902 Zeeman received with Lorentz his Nobel Prize.

\section{Einstein's Relativity: Is Ether a Propagation Medium for the Velocity of Light?}

Following my reading of E.'s original contributions, I believe that the popular understanding of E.'s decision to abolish ether in his celebrated 1905 essay on special relativity needs deeper historical and philosophical analysis. E.'s ideas on the ether problem are in fact strictly related to his technical and philosophical approaches to the whole context of his revolutionary relativity theories. E.'s original ideas on ether are condensed in one of his important, but less quoted essay, devoted to his early discussions on ether in his 1920 book Relativity, the Special and General Theory ${ }^{36}$, and to his return to the same problem in his 1954 fifth Appendix added to the book, and titled: "Ether and the Theory of Relativity and the Problem of Space”. Let us resume E.'s most interesting remarks on ether in his 1920 publication. The "far-reaching similarity, which subsists between the properties of light and those of elastic waves in ponderable bodies", represented in E.'s view "a fresh support”, for an elastic type of ether as a medium for Light's waves. But the elastic approach to theory was also a source of great difficulties, because "neither Maxwell nor his followers succeeded in elaborating a mechanical model for the ether which might furnish a satisfactory mechanical interpretation of Maxwell's laws of the electro-magnetic field. The laws were clear and simple, the mechanical interpretations clumsy and contradictory" (My Italics) ${ }^{37}$. In E.'s view, his special theory of relativity overcame the difficulties by an hard restriction. In his essay he assumed that ether consisted of particles whose motion was not observable through time: "There may be supposed to be extended physical objects to which the idea of motion cannot be applied... The special theory of relativity abstracted from ether the last mechanical characteristic. But, a non mechanical ether might appear as a superfluous requirement in place of electromagnetic fields as ultimate, irreducible realities...If from the standpoint of ether this hypothesis appears at first to be an empty hypothesis, one should consider that in the electromagnetic processes in vacuo... the electromagnetic fields appear as ultimate, irreducible realities, and at first it seems superfluous to postulate a homogeneous, isotropic ether-medium, and to envisage electromagnetic fields as states of this medium". As unexpected as it might seem, this hypothesis aims to reconcile the two contrasting position of a mechanical or an electromagnetic state of ether, because Einstein does not renounce to ether: "on the other hand there is a weighty argument to be adduced in favor of the ether hypothesis. To deny the ether is ultimately to assume that empty space has no physical qualities whatever. The fundamental facts of mechanics do not harmonize with this view". Surprising enough, Newton's authority is still called in support of a physical conception of space. According to Einstein in the general tendency to give conceptual priority to electromagnetic concepts

\footnotetext{
${ }^{35}$ Zueeman, P (1967).

${ }^{36}$ Einstein, A. (1922) Einstein gave an address on 5 May 1920 at the University of Leiden. He chose as his topic Ether and the Theory of Relativity. He lectured in German but we present an English translation below. The lecture was published by Methuen \& Co. Ltd., London, in 1922.

${ }^{37}$ Einstein, A. (1922): p. 18.
} 
sought a solution to the ether problem, by reducing the principles of mechanics to those of electricity: "especially as a confidence in the strict validity of the equations of Newton's mechanics was shaken by the experiments with radioactive-rays and rapid cathode rays". A positive influence for this solution, represented by the electro-dynamical investigations of Heinrich Hertz, was neglected by Einstein because "the dualism still confronts us... in the theory of Hertz, where matter appears not only as the bearer of velocities, kinetic energy, and mechanical pressures, but also as the bearer of electromagnetic fields”.

E.'s approach to ether couldn't disregard his relation with Lorentz, the authoritative supporter of an ethereal reality. Einstein tends to conciliate the contrasting feature of the Lorentian approach: "The space-time theory and the kinematics of the special theory of relativity were modeled on the Maxwell-Lorentz theory of the electromagnetic field”. Moreover: “According to Lorentz's theory, electromagnetic radiation, like ponderable matter, brings impulse and energy with it, and as, according to the special theory of relativity, both matter and radiation are but special forms of distributed energy, ponderable mass losing its isolation and appearing as a special form of energy"38.

The role of a new ether was part of E.'s life interest, and of his recurrent hope of an unification of electromagnetism and gravitation. Einstein's remarks in the same essay are thus presented: “As to the part which the new ether is to play in the physics of the future we are not yet clear. We know that it determines the metrical relations in the space-time continuum, e.g., the configurative possibilities of solid bodies as well as the gravitational fields; but we do not know whether it has an essential share in the structure of the electrical elementary particles constituting matter. Nor do we know whether it is only in the proximity of ponderable masses that its structure differs essentially from that of the Lorentzian ether; whether the geometry of spaces of cosmic extent is approximately Euclidean”.

In his General Relativity in fact ether was accepted, as long as its physical nature could be conceived as something different from the centuries old concept of an uniform distributed special substance: "What is fundamentally new in the ether of the general theory of relativity as opposed to the ether of Lorentz consists in this, that the state of the former is at every place determined by connections with the matter and the state of the ether in neighboring places, which are amenable to law in the form of differential equations; whereas the state of the Lorentzian ether in the absence of electromagnetic fields is conditioned by nothing outside itself, and is everywhere the same. The ether of the general theory of relativity is transmuted conceptually into the ether of Lorentz if we substitute constants for the functions of space, which describe the former, disregarding the causes, which condition its state. Thus we may also say I think, that the ether of the general theory of relativity is the outcome of the Lorentzian ether, through relativation" (Italics S.D).

The theme of a theory that could succeed in associating gravitational and electromagnetic field as one unified conformation, interested Einstein's research on a Generalized Field Theory for the rest of his life. In this connection, E.'s early neglect of ether declines in his mature reflections, and more comprehensive considerations are consistent with the fundamental tenets of his general relativity: "More careful reflection teaches us however, that the special theory of relativity does not compel us to deny ether. We may assume the existence of an ether; only we must give up ascribing a definite state of motion to it, i.e., we must by abstraction take from it the last mechanical characteristic which Lorentz had still left it. We shall see later that this point of view... is justified by the results of the general theory of relativity". This view of his ether is to be regarded as his last conviction in the closing years of his life: "Recapitulating, we may say that according to the general theory of relativity space is endowed with physical qualities; in this sense, therefore, there exists an ether. According to the general theory of relativity space without ether is unthinkable; for in such space there not only would be no propagation of light, but also no possibility of existence for standards of space and time (measuring-rods and clocks), nor therefore any space-time intervals in the physical sense. But this ether may not be thought of as endowed with the quality characteristic of ponderable media, as consisting of parts which may be tracked through time. The idea of motion may not be applied to it.”39

\section{Concluding Remarks}

The problem of the nature of Light and of the role of Ether, as its medium or source, was very central in the scientific literature at the end of the Nineteenth Century, and thus one may take account for the German and

\footnotetext{
${ }^{38} \mathrm{Ibid}, 147$.

${ }^{39}$ Ibid, 147. E. never lost faith in a conciliation of GR with Quantum Theories. Let us consider his conclusive statement: "Further, in contemplating the immediate future of theoretical physics we ought not unconditionally to reject the possibility that the facts comprised in the quantum theory may set bounds to the field theory beyond which it cannot pass".
} 
European scientists' reaction to E.'s abolition in his 1905 essay. A short and approximate list of his intellectual opponents cannot miss to mention Mach's indifference, Poincaré and the French culture silence, not to mention the harsh and partially motivated criticism of colleagues in E.'s own country. Following J.J. Thomson's discovery, physicist considered the oscillating electrons as the Light's physical source. Were electrons performing the role of the old-fashioned Keplerian Lumen? The demand can evidently be answered on a physical level, either on a cosmological basis, as by a Big Bang type modern theory, or by the black matter mysterious presence. In this paper, I argued, however, that such questions also deserved an historical and philosophical approach. As for an example, although Helmholtz' and Maxwell's theories were conditioned by their technical expertise, their arguments concerned the philosophical problem of perceptions and its bearing on the nature of Light. Let us consider in fact Hertz's case on the discovery of Maxwell's waves. I noticed a remarkable interdependence between the final Hertz's representation of the waves and the philosophical conception of ether that was a background to his discovery. The discovery corresponded to the moment when he realized that the conception of a dielectric “a la Poisson” was not sufficient to support the conviction of the existence of waves of electric force in air, but that their existence had to be conceived as a primitive polarization of ether. The discovery was in some way a consequence of this change of conceptions and strengthened, in turn, this change. I consider this example as a very relevant instance of the strength of preconceived, i.e., a-priori ideas in physics research.

Although the study of Light limited to its visual properties represented the bases themselves of the initial science of optics, and of the nature of Light, the discovery of electromagnetic non optical of X rays had the rather anomalous effect of diminishing the intrinsic interest for the nature of Light. It was replaced by an increased research on similarities within the extended band of electromagnetic waves, whose whole spectrum covered several millions of units Hertzes, whereas the visible section included just three hundreds Hertzcs.

No wonder that in this situation Helmholtz represented one of the leaders in middle of Century in physical and physiological optics. By his regularity Principle he hardly tried to discriminate between physical and physiological principles. In my view, his thought stands on the watershed between two great traditions in the development of modern physics. On the one hand, through the attention given to perceptions per se and psychology, he opened the way to Mach's phenomenology. On the other hand, with his special upholding of Kant's a-priori, he favored Hertz's philosophy and Hertz's particular re-evaluation of Kant's a-priori.

Passionate discussions among physicists were very frequent in the same period of Einstein's intervention. But the historian Rosenfeld rightly remarked that one of the effects of Hertz's great experiment was to deviate the major line of discussion from the ether's to the problem of the evident similarity between the well known properties of Light, and that of the recently produced electromagnetic waves of various frequencies (X rays included). Their experimental production had the effect of polarizing the physicist's interest, relinquishing to a secondary level the problem of their nature and of their relation with ether ${ }^{40}$.

On the other side, the international success of Maxwell's and Hertz's local field theories, and the almost cotemporary triumph of Lorentz's and Zeeman's antagonist theories of an atomistic microphysics, required a new type of analysis to scientists, historians, and philosophers of science. Lorentz, the best representative of the recently developed particles and waves Electrodynamics, frequently expressed reactions to the ether abolition. Although ontological determinism, realism, and purely phenomenological positions, were "a la pair”, Einstein did not regard his decision as one of his alleged antirealist approaches to physics ${ }^{41}$.

It was Einstein's merit in 1905 to perceive that excluding Ether in his Special Relativity would advantage theoretical physics. However, he did not consider its exclusion as an apodictic decision. At the end of his scientific life he was still fundamentally convinced of the indispensability: "According to the general theory of relativity space without Ether is unthinkable: for in such a space there would not only be no propagation of light but also no possibility of existence for standards of space and time”42. E. never lost faith in a conciliation of GR with Quantum Theories. Let us consider his conclusive statement: "Further, in contemplating the immediate future of theoretical physics we ought not unconditionally to reject the possibility that the facts comprised in the quantum theory may set bounds to the field theory beyond which it cannot pass”.

Let us conclude: E.'s decision in 1905 to abolish ether in his special relativity did not settle the problem once for all. Let me try to condense in a few drastic sentences Einstein's alleged doubts in front of his dramatic decision. If one accepts (pace Maxwell and Hertz) that Light is electricity, and that ether is analogous to electricity

\footnotetext{
${ }^{40}$ Rosenfeld (1956).

${ }^{41}$ Howard, D. (1993).

${ }^{42}$ Einstein, A. (1922).
} 
itself (pace Hertz’s dielectric ether), how might one abolish ether without abolishing electricity? Einstein's solution consisted in extracting from ether the old fashioned ethereal attributes, of substance and motion, and leaving apart a few properties listed in his considerations. It is thus comprehensible that he often returned to arguments concerning his logical and epistemological justification. They were variously motivated, but on the whole, let me allow to drastically resuming two Einstein's divergent positions. Accepting the role of ether as a material medium for transmission of electromagnetic waves and of Light presented the great difficulty of theoretically justifying the enormous high value of Light's velocity, whereas any attempt at an elastic type form of mechanical explanation was by him definitively rejected. On the other side, as shown above, he was reluctant to view the magnetic and electric fields as entities independently existing and moving with a specific velocity in a vacuum space-time. From an historical perspective, the Einstein's time conceptual relationship among ether, Light, and elctromagnetic waves, was very far from presenting hopes for an axiomatic rational approach. Einstein settled the problem in his General Relativity by stating that in line of principle the velocity of Light was a universal constant of nature.

\section{References}

Bartellini, F. (2010). La scomparsa della sfera del fuoco ela nascita del fuoco d'amore. Novi Ligure: Edizioni Joker.

Cassirer, E. (1950). The Problem of Knowledge, Philosophy of Science and History of Science since Hegel. New Haven, CT: Yale University Press.

D’Agostino, S. (1975). Hertz's Researchers on Electromagnetic Waves. Historical Studies in the Physical Sciences, 6, 261323. http://dx.doi.org/10.2307/27757343

D’Agostino, S. (1996). Absolute Systems of Units and Dimensions of Physical Quantities. Physis (Rivista Internazionale di Storia della Scienza), XXXIII, Fasc. 1-3, NS.

Einstein, A. (1922). Ether and Relativity. Sidelights on Relativity. Trans by Lawson, R. W. London: Henry Holt and Company.

Hendry, J. (1986). James Clerk Maxwell and the Theory of Electroamgnetic Field. Bristol and Boston: Adam Hilger Ltd.

Hermann von Helmholtz (1977). Epistemological Writings: The Paul Hertz/Moritz Schlick Centenary Edition of 1921, with Notes and Commentary by the Editor (Ed. with Introd. and Bibliography by R. S. Cohen, \& Y. Elkana). Boston Studies of the Philosophy of Science, Vol. 37. Dordrecht: Springer. http://dx.doi.org/10.1007/978-94-010-1115-0

Hertz, H. (1956a).The Principles of Mechanics Presented in a New Form. Prefase of H.von Helmholtz.Autorized English Translation by Jones D.R. and Walley J.T. with a new Introduction by Cohen R.S. New York: Dover Pbl. Inc.

Hertz, H. (1956b). On the Relation between Maxwell's Fundamental Electromagnetic Equations and the Fundamental Equations of the Opposing Electromagnetics. Miscellaneous Papers. London: Macmillan. English Translation of Hertz, H. (1884) Schriften vermischten Inhalts.Gesammelte Werke, Band I. Leipzig: J.A. BarthVerlag.

Hertz, H. (1962). Electric Waves, Being Researches on the Propagation of Electric Action with Finite Velocity through Space. New York: Dover Pbl Inc. Authorized English Translation by Jones D.E. of Hertz, H. (1892). Untersuchungen ueber die Ausbreitung der elektrischen Kraft. Leipzig: Johann Ambrosius Barth.

Howard, D. (1993). Was Einstein Really a Realist? Perspectives on Science: Historical, Philosophical, Social, 1, $204-251$.

Hunt, B. J. (1991) The Maxwellians. Cornell History of Science Series.

Lorentz, H. A. (1952). Poblems of Modern Physics. New York: Dover.

Maxwell, J. C. (1954) The Scientific Papers of James Clerk Maxewll. Two Volumes. New York: Dover Publications.

Planck, M. (1931). James Clerk Maxwell in seine Bedueting fuer die Theoretische Physik in Deutschland. Naturwiss, 18891894.

Ronchi, V. (1952). Storia della Luce. Fondazione “Giorgio Ronchi”. Second Edit. Zanichelli, Bologna: Casa Edit.

Rosenfeld, L. (1956). The Velocity of Light and the Evolution of Electrodynamics. Il Nuovo Cimento, 4, 1630-1667. http://dx.doi.org/10.1007/BF02745315

Zeeman, P. (1967) Light Radiation in a Magnetic Field. Nobel Lectures. Physics 1901-1921. Amsterdam: Elzevier Publ. Co. 